Revista Brasileira de

Engenharia Agrícola e Ambiental

v.15, n.11, p.1193-1198, 2011

Campina Grande, PB, UAEA/UFCG - http://www.agriambi.com.br

agriambi

Protocolo 246.10 - 31/12/2010 • Aprovado em 05/09/2011

\title{
Análise termográfica da temperatura superficial de telhas
}

\author{
Paulo G. de Abreu ${ }^{1}$, Valéria M. N. Abreu ${ }^{1}$, Arlei Coldebella ${ }^{1}$, \\ Letícia dos S. Lopes ${ }^{1}$, Vanessa da Conceição ${ }^{2}$ \& Inaiara L. Tomazelli ${ }^{3}$
}

\section{RESU M O}

N este trabalho objetivou-se realizar imagens termográficas da superfície de telhas visando avaliar sua temperatura superficial. O s tratamentos mensurados se constituíram de: T1 - Telha Coppo Venneto Ondulada Cinza; T2 - Telha Coppo Venneto O ndulada Marfim; T3 - Telha Plana Marfim; T4 - Telha Plana Cinza; T5 - Telha de Barro Colonial esmaltada; T6 - Telha O ndulada de Cimento Amianto e T7 Telha de Barro Francesa. Cada unidade experimental foi composta de três telhas sobrepostas uma sobre a outra, exceto no tratamento de telha ondulada de cimento amianto. 0 delineamento experimental foi em blocos casualizados com três repetições. Os dados coletados em cada horário foram analisados por meio do modelo de medidas repetidas, utilizando-se o procedimento MIXED do SAS. As temperaturas da superfície inferior das telhas foram muito parecidas ao longo do dia. As telhas com coloração marfim obtiveram melhores valores de temperatura superior e inferior que as telhas com coloração cinza, nas respectivas categorias. A telha de barro colonial obteve maior temperatura da superfície inferior que as demais telhas.

Palavras-chave: ambiência animal, conforto térmico, tipos de cobertura, telhado

\section{Thermographic analysis of the superficial temperature of roof tiles}

\begin{abstract}
The objective of study was to get thermographic images from the surface of roof tiles to evaluate their tempertures. The evaluated treatments were: T1 - grey Coppo Venneto wave tiles; T2 -Ivory-colored Coppo Venneto wave tiles; T3 - Ivory- colored, plain tile; T4 - plain, grey roof tile; T5 - glazed clay colonial tile; T6 - Asbestos wave tile T7 - French clay tiles. Each treatment was made up of three tiles which were piled one top of the other, except for the asbestos tiles. The experimental design was in randomized block with three repetitions. The data which were collected in each set time were analyzed through the repetitive measure model, using the MIXED procedure - SAS. The temperatures of the inferior surface of the tiles were very similar throughout the day. The ivory colored tiles had better superior and inferior temperature than the grey-colored ones in their respective category. The glazed, clay colonial tile presented a larger temperature of inferior surface than the other tiles.
\end{abstract}

Key words: animal well-being, thermal comfort, types of roof coverage, roofs

\footnotetext{
Embrapa Suínos e Aves, BR 153, KM 110, CEP 89700-000 Concórdia, SC. Fax: (49) 3441.0497; Fone: (49) 3441-0400. E-mail: pabreu@cnpsa.embrapa.br; valeria@cnpsa.embrapa.br; arlei@cnpsa.embrapa.br; leticia@cnpsa.embrapa.br

2 Graduanda de Engenharia Ambiental, U niversidade do Contestado (U nC). Bolsista CN Pq. Rua Victor Sopelsa 3000, Bairro Salete, CEP 89700-000, Concórdia, SC. Fone (49) 3441.1000. E-mail: vanessa@hotmail.com

${ }^{3}$ G raduanda em Ciências Biológicas, U niversidade do Contestado (UnC), Rua Vitor Sopelsa 3000. Bairro Salete. CEP 89700-000. Concórdia, SC. Fone (49) 3441.1000. E-mail: ina_It@yahoo.com.br
} 


\section{INTRODUÇÃO}

No Brasil, país de clima tropical com temperaturas elevadas de verão e intensa radiação, os materiais a serem utilizados para a confecção das coberturas devem permitir bom isolamento térmico para que o ambiente interno das instalações seja menos influenciável pela variação climática (Abreu et al., 2001).

As limitações para obtenção de altos índices zootécnicos no Brasil decorrem do alojamento em ambientes com clima quente, de animais geneticamente desenvolvidos em climas mais amenos, em condições ou conceitos provenientes deste clima; daí a necessidade de se ter instalações adaptadas com características construtivas que garantam o máximo possível de conforto permitindo, ao animal abrigado, desenvolver todo o seu potencial genético (Fiorelli et al., 2009).

Para Turnpenny et al. (2000) em instalações de animais o telhado tem papel primário na determinação das suas trocas térmica, principalmente em regiões com clima quente.

Assim, o telhado das instalações tem sido o elemento mais relevante a ser considerado para se promover o conforto térmico dos animais em regiões de clima quente razão por que em algumas regiões do mundo tem sido utilizado como elemento para reduzir o ganho de calor total da telha, provendo efeito refrescante para as instalações (Faghih \& Bahadori, 2009).

O telhado dispõe de condições para resfriar as instalações mas se ele não executa bem esta função poderá apresentar problemas enormes às instalações. Este é o elemento da instalação mais exposto ao céu e quase metade do calor ganho é através dele. Por outro lado, o telhado abre uma gama extensiva de possibilidades para dissipar o calor da instalação (Tang \& Etzionb, 2005).

Em regiões com clima quente, alta resistência térmica nas horas mais quentes do dia pode reduzir o efeito da radiação solar poém, aumentando a resistência térmica, poderá reduzir a transferência de calor do telhado no período noturno, que é mais fresco (Liberati \& Zappavigna, 2007).

A baixa temperatura superficial do telhado leva a também uma baixa condução de calor para o interior da instalação (Akbari et al., 2005). A energia solar absorvida ocasiona aumento da temperatura do telhado em comparação com a temperatura do ar ambiente (Faghih \& Bahadori, 2010)

A utilização de materiais refletivos à radiação solar traz benefícios econômicos por permitir a redução do ganho de calor pelos telhados visto que uma cobertura exposta ao sol atua como coletor de energia solar (Wray \& Akbari, 2008).

O material ideal para a telha deve atender às recomendações com as quais a superfície superior tenha alta refletividade solar e alta emissividade térmica e a superfície inferior tenha baixa refletividade solar e baixa emissividade térmica (Abreu et al., 2001).

D'Orazio et al. (2010) mostraram que o aumento da densidade de isolamento do telhado reduz as estratégias tradicionais de resfriamento artificial no interior das instalações.

A cobertura mais utilizada nas instalações avícolas brasileiras é a de cimento amianto, por apresentar facilidades de instalação, manutenção e limpeza, além do custo inferior, quando comparada com telhas de barro e alumínio (Moraes et al., 1999). Essas ainda podem ser associadas a outros métodos para diminuir a temperatura dentro da instalação, tais como pintura reflexiva e aspersão na face externa.

Melhorias adicionais no ambiente térmico interno de instalações podem ser conseguidas com associação das coberturas tradicionais com materiais isolantes, reflexivos e/ ou de grande inércia térmica, uso de forros variados sob a cobertura e pintura com pigmentos isolantes e/ou reflexivos sobre e/ou sob as telhas (Moraes et al., 1999).

Pelo exposto, objetivou-se realizar imagens termográficas da superfície de telhas, visando avaliar sua temperatura superficial.

\section{Material e MÉTOdos}

O experimento foi realizado no Centro Nacional de Pesquisa em Suínos e Aves (Embrapa CNPSA) - Concórdia, SC, que se encontra na latitude de $27^{\circ} 18^{\prime} 51,92$ "S e longitude de $51^{\circ} 59^{\prime}$ 44,48 " W e a $538 \mathrm{~m}$ de altitude.

Os tratamentos avaliados se constituíram de sete tipos de telha, a saber: T1 -Telha Coppo Venneto Ondulada Cinza; T2 Telha Coppo Venneto Ondulada Marfim; T3 - Telha Plana Marfim; T4 - Telha Plana Cinza; T5 - Telha de Barro Colonial esmaltada; T6 - Telha Ondulada de Cimento Amianto-0,006 m e T7 - Telha de Barro Francesa.

As telhas Coppo onduladas eram de concreto com tamanho médio de 0,42 x 0,33 m e peso de 4,9 $\mathrm{kg}$ e as Planas eram do mesmo material e tamanho, com peso de $5,10 \mathrm{~kg}$.

As telhas foram colocadas ao ar livre, em estrutura de madeira, em uma água com inclinação de $7^{\circ}$ e distante do piso a um metro, voltada para o sul e orientada no sentido lesteoeste.

Cada unidade experimental foi composta de três telhas sobrepostas uma sobre a outra, exceto no tratamento de telha cimento amianto em que o comprimento era de $1,53 \mathrm{~m}$. O delineamento experimental foi em blocos casualizados, com três repetições (Figura 1).

\begin{tabular}{|c|c|c|c|c|c|c|c|c|c|c|c|c|c|c|c|c|c|c|c|c|c|c|c|}
\hline \multicolumn{10}{|c|}{} & \multicolumn{1}{|c|}{ Bloco 2} & \multicolumn{1}{|c|}{ Bloco 3 } \\
\hline $\mathrm{T}$ & $\mathrm{T}$ & $\mathrm{T}$ & $\mathrm{T}$ & $\mathrm{T}$ & $\mathrm{T}$ & $\mathrm{T}$ & $\mathrm{T}$ & $\mathrm{T}$ & $\mathrm{T}$ & $\mathrm{T}$ & $\mathrm{T}$ & $\mathrm{T}$ & $\mathrm{T}$ & $\mathrm{T}$ & $\mathrm{T}$ & $\mathrm{T}$ & $\mathrm{T}$ & $\mathrm{T}$ & $\mathrm{T}$ & $\mathrm{T}$ & $\mathrm{T}$ & $\mathrm{T}$ & $\mathrm{T}$ \\
\hline & 3 & 6 & 4 & 1 & 5 & 7 & 8 & 5 & 7 & 8 & 1 & 4 & 6 & 3 & 2 & 4 & 6 & 7 & 2 & 8 & 1 & 5 & 3 \\
\hline
\end{tabular}

Figura 1. Esquema da distribuição dos tratamentos na unidade experimental

As imagens reais e termográficas das superfícies superior e inferior das telhas foram coletadas a cada três horas, de 0 a 24 horas, por meio de um termovisor TESTO 880.

O termovisor (sensibilidade térmica $<0,1^{\circ} \mathrm{C}$, emissividade térmica de 1 (globo negro ideal), espectro de - $14 \mathrm{~mm}$ ) possuindo internamente câmera digital integrada, foi conectado a uma sonda de umidade e temperatura, via rádio frequência RFID registrando os valores de temperatura ambiente.

O programa computacional Texto IRSoft, foi utilizado para traduzir o espectro de cores da medida da temperatura. A partir das imagens termográficas das telhas foram delineados os perímetros correspondentes e determinados os valores máximo, mínimo e médio de temperatura da superfície inferior e superior (Figura 2). 

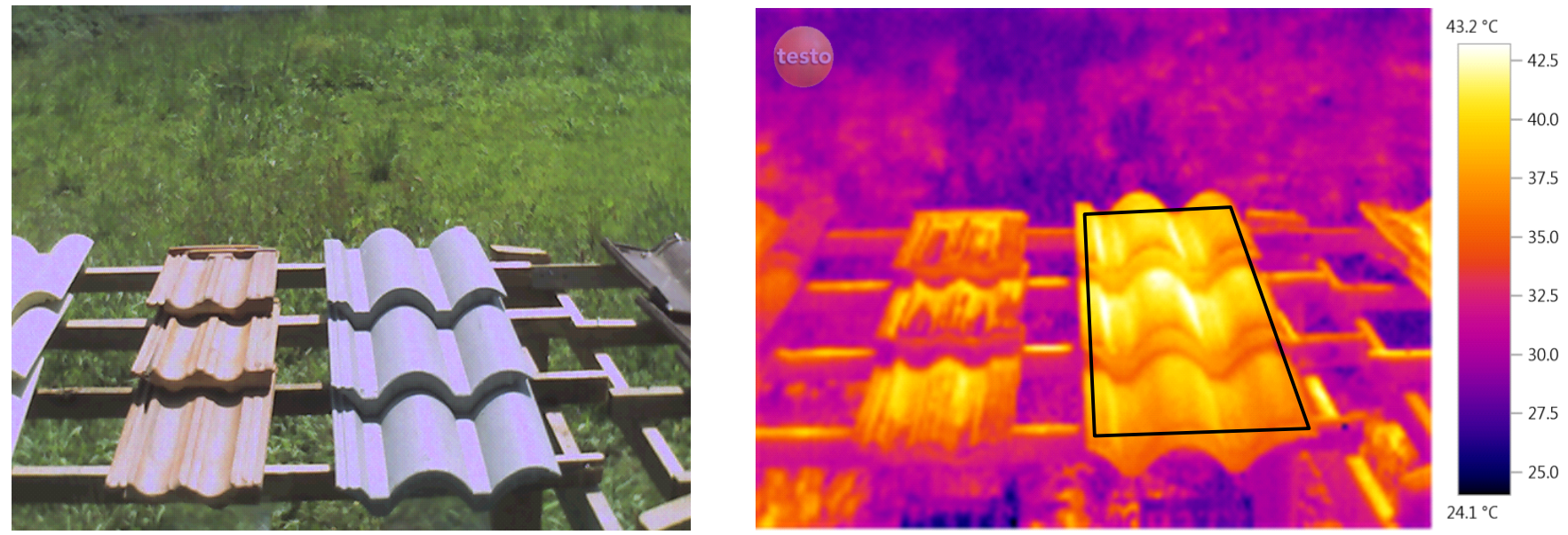

Figura 2. Imagem real e termográfica com o perímetro de medida das temperaturas

Os dados coletados em cada horário foram analisados por meio do modelo de medidas repetidas, utilizando-se o procedimento MIXED do SASÔ, e testadas 15 estruturas de variância e covariância, escolhendo-se a que apresentou menor valor para o Critério de Informação de Akaike (AIC).

Foram testados os efeitos de bloco, tratamento (telhas), hora e a interação entre tratamento e hora. $\mathrm{O}$ desdobramento dos efeitos foi realizado por meio do teste $t$.

Para analisar os dados de temperaturas médias das telhas (superior e inferior) foi usada a análise harmônica, conforme Morettin \& Toloi (2004), por meio do modelo descrito na Eq. (1):

$$
\mathrm{y}_{\mathrm{t}}=\mu+\mathrm{R} \cos (\omega \mathrm{t}+\phi)+\varepsilon_{\mathrm{t}}
$$

sendo:

$y_{t}$ - valor observado das variáveis no tempo $t$

$\mu$ - média; $R$, a amplitude

$\phi$ - o ângulo de fase

$\omega$ - frequência, no caso fixa, em $\pi / 12$ e $\varepsilon_{\tau}$, a componente aleatória

Para facilitar a estimação dos parâmetros do modelo da Eq. (1), foi ajustado o modelo a Eq. (2) equivalente a:

$$
y_{t}=\mu+A \cos (\omega t)+B \operatorname{sen}(\omega t)+\varepsilon_{t}
$$

sendo que:

$$
R=\sqrt{A^{2}+B^{2}}
$$

$\mathrm{e}$

$$
\phi=\operatorname{arctg}(-\mathrm{B} / \mathrm{A})
$$

No intuito de verificar o efeito dos tratamentos sobre os parâmetros $\mu, \operatorname{Re} \phi$, as estimativas seus efeitos foram calculados para cada unidade experimental. A partir dessas estimativas foram avaliados os efeitos de bloco e tratamento sobre parâmetros $\mu, \mathrm{R}$ e $\phi$, utilizando-se a análise de variância, por meio do PROC GLM do SAS (2003).
O desdobramento dos efeitos foi realizado por meio do teste $\mathrm{t}$.

\section{RESULTADOS E DISCUSSÃO}

A interação Tratamento $\mathrm{x}$ Hora foi significativa para todas as variáveis $(\mathrm{p}<0,05)$.

A condição essencial para a transmissão de calor é que haja diferença de temperatura entre os meios, denominado gradiente de temperatura. $\mathrm{O}$ gradiente de temperatura indica o sentido do fluxo de calor. O sentido sempre vai ocorrer do maior para o menor valor. Se as temperaturas dos meios forem iguais não haverá transferência de calor, caso em que se diz que os meios estão em estado de equilíbrio.

Assim, na Figura 3 o sentido do fluxo de calor foi negativo e ascendente, ou seja, da superfície inferior para a superior da telha, em todo o período, para todos os modelos de telha avaliados, exceto a telha de amianto no período de 15 e $18 \mathrm{~h}$, significando que as telhas na superfície inferior aqueceram mais que na superfície superior.

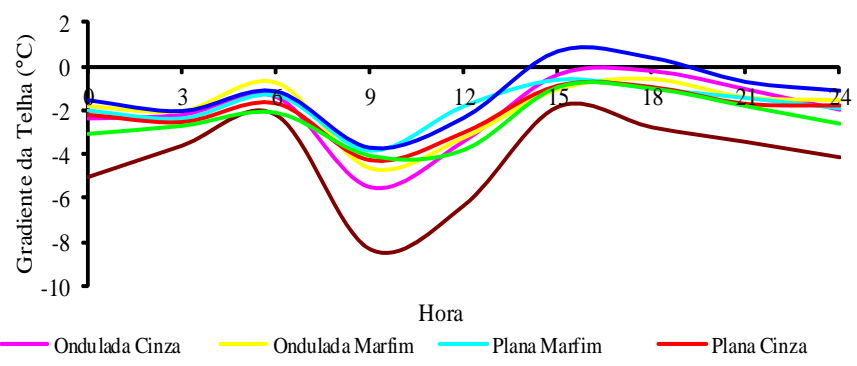

- Barro Colonial - Amianto Francesa

Figura 3. G radiente de temperatura entre a superfície superior e inferior da telha $(\mathrm{Gt}=\mathrm{Ts}-\mathrm{Ti}) \mathrm{Gt}=$ gradiente da telha Ts = Temperatura da superfície superior da telha $\mathrm{Ti}=$ Temperatura da superfície inferior da telha

No interior das telhas a transferência de calor ocorre por meio da condução realizada por contato entre as moléculas ou partículas das telhas. Para determinar a quantidade de calor transmitida é necessário conhecer o coeficiente térmico de condução (K) e a espessura do material. Às 15 h, a direção do fluxo de calor foi descendente para a telha de amianto (Figura 
3). Neste contexto, o fluxo de calor através das coberturas, juntamente com as elevadas temperaturas na face inferior das telhas, é a causa principal do desconforto no interior das instalações (Rosa, 1984).

Este fato é ruim para os sistemas de criação de aves e suínos, uma vez que a energia térmica da superfície superior da telha é repassada para a superfície inferior da telha podendo elevar a temperatura interna da instalação, nas horas de maior estresse por calor.

A transmissão do calor entre a telha e o ar ambiente tem efeito da movimentação do ar. O processo possui duas fases: na primeira, o calor se transmite por contato ou por condução; na segunda, a alteração sofrida pela temperatura do ar modifica sua densidade fazendo com que haja um movimento convectivo ascendente ou descendente.

$\mathrm{O}$ gradiente de temperatura médio superior entre o ar e a superfície superior da telha foi positivo em todos os modelos de telha, entre os horários de $0,3,6,19$ e 21 h, mostrando que o sentido do fluxo de calor nesse período é do ar para a telha (Figura 4).

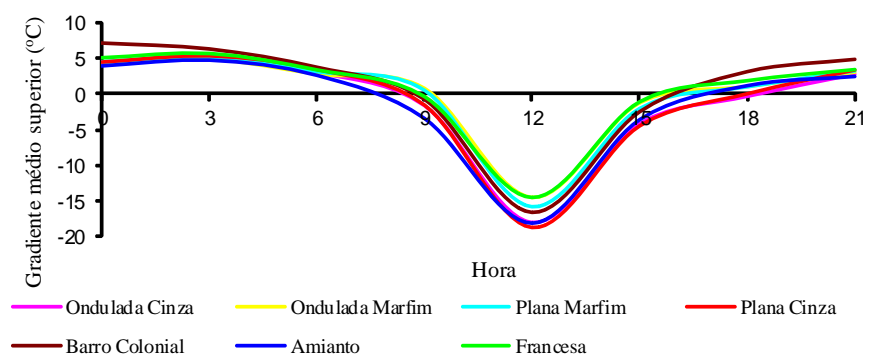

Figura 4. Gradiente médio da temperatura entre o ambiente e a temperatura média da superfície superior da telha ( $\mathrm{G}$ meds $=\mathrm{Ta}$ - Ts), G meds - gradiente médio superior; Ta - temperatura ambiente; Ts - temperatura da superfície superior da telha

O gradiente de temperatura foi, às $12 \mathrm{~h}$, negativo para todas as telhas. Neste horário, sendo o valor do gradiente negativo significa que o sentido do fluxo de calor será da telha para o ar (Figura 4). A cor branca da superfície externa do telhado de amianto foi eficiente na redução da temperatura da superfície interna da cobertura, reduzindo em até $9,0^{\circ} \mathrm{C}$ a temperatura, no horário das 13 horas (Sarmento et al., 2005).

$\mathrm{Na}$ superfície inferior das telhas os gradientes tiveram o mesmo comportamento cíclico sendo que, às $12 \mathrm{~h}$, apresentaram variação no valor (Figura 5). Furtado et al. (2003) analisaram galpões cobertos com telha cerâmica e amianto e verificaram que os valores de carga térmica de radiação sob coberturas de telha de amianto são superiores aos de telha cerâmica.

Quando a energia radiante incide sobre um corpo, ela é absorvida, refletida e transmitida (Figura 6). A energia absorvida se transforma em energia térmica ou calor mas a radiação refletida e a transmitida não sofrem modificação alguma.

Na Tabela 1 verifica-se efeito de tratamento para os três parâmetros, nas duas variáveis analisadas $(\mathrm{p}<0,05)$.

As telhas com coloração marfim obtiveram melhores valores de temperatura superior e inferior que as telhas com coloração cinza, nas respectivas categorias (Tabela 2).

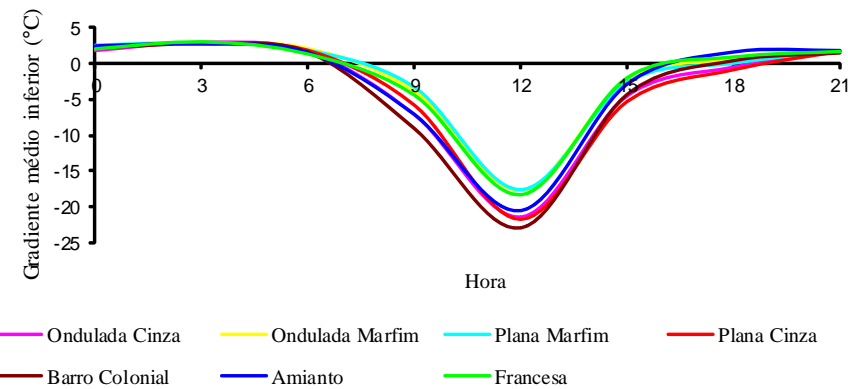

Figura 5. Gradiente médio da temperatura entre 0 ambiente e a temperatura média da superfície inferior da telha $(\mathrm{Gmedi}=\mathrm{Ta}-\mathrm{Ti})$, Gmedi - gradiente médio inferior; $\mathrm{Ta}$ - temperatura ambiente; $\mathrm{Ti}$ - temperatura da superfície inferior da telha

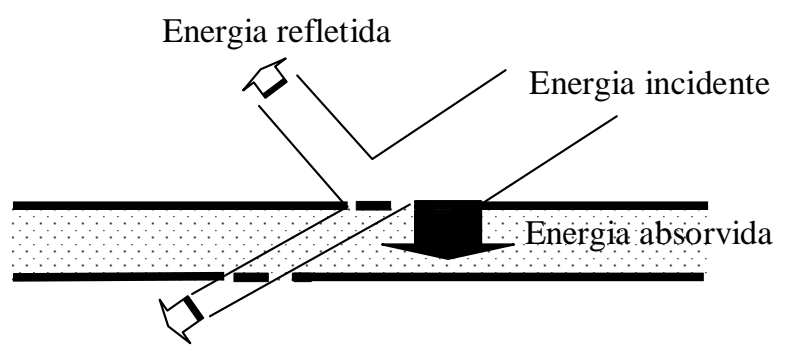

Energia transmitida

Figura 6. Transferência de calor em uma superfície

Tabela 1. Níveis descritivos de probabilidade do teste $F$ da análise da variância para os parâmetros $\mu$, R e $\phi$ das temperaturas médias, inferior e superior

\begin{tabular}{|c|c|c|c|c|c|c|}
\hline \multirow{3}{*}{$\begin{array}{l}\text { Causa de } \\
\text { variação }\end{array}$} & \multicolumn{6}{|c|}{$\operatorname{Pr}>\mathrm{F}$} \\
\hline & \multicolumn{3}{|c|}{ Temperatura média inferior } & \multicolumn{3}{|c|}{ Temperatura média superior } \\
\hline & $\mu$ & $\mathbf{R}$ & $\phi$ & $\mu$ & $\mathbf{P}$ & $\phi$ \\
\hline Bloco & 0,6205 & 0,0003 & 0,0064 & $0,0145<$ & 0,0001 & 0,0823 \\
\hline Tratamento & 0,0001 & 0,0001 & 0,0001 & $<0,0001<$ & 0,0001 & 0,0001 \\
\hline
\end{tabular}

Sarmento et al. (2005) avaliaram a influência da pintura externa do telhado sobre a temperatura da superfície interna da telha, o acondicionamento térmico do galpão e o desempenho produtivo de frangos de corte em galpões cobertos com telha de fibrocimento e ventilação artificial, sendo um com as telhas pintadas de branco e o segundo na forma natural e concluíram que as telhas pintadas de branco foram mais eficientes na redução da temperatura da superfície interna da cobertura.

A forma ondulada ou plana não influenciou nos valores de temperatura das telhas. A telha de barro colonial obteve maior amplitude e temperatura da superfície inferior que as demais telhas. As temperaturas médias da temperatura superior das telhas ondulada cinza, plana cinza e amianto, foram maiores que nas demais telhas, não havendo diferença significativa entre as mesmas.

Santos et al. (2002) analisaram coberturas com telhas de barro e alumínio, comumente utilizadas em instalações animais, para duas alturas distintas de pé-direito, em condições de inverno, e concluíram que nas horas de frio mais intenso todas as coberturas causaram desconforto térmico e todos os protótipos tiveram umidade relativa acima do máximo tolerável para o conforto animal de inverno, no Brasil. 
Tabela 2. M édias e erros-padrão por tratamento e parâmetro para a temperatura média inferior e superior da telha

\begin{tabular}{crrrrrrr}
\hline \multirow{2}{*}{ Parâmetro } & \multicolumn{7}{c}{ Tratamentos } \\
\cline { 2 - 7 } & Ondulada Cinza & Ondulada Marfim & Plana Marfim & Plana Cinza & Barro Colonial & Amianto & Francesa \\
\multicolumn{7}{c}{ Temperatura média inferior } \\
$\mu$ & $26,160 \pm 0,050 \mathrm{~B}$ & $24,710 \pm 0,040 \mathrm{E}$ & $24,710 \pm 0,030 \mathrm{E}$ & $26,100 \pm 0,030 \mathrm{~B}$ & $26,480 \pm 0,030 \mathrm{~A}$ & $25,530 \pm 0,070 \mathrm{C}$ & $24,910 \pm 0,020 \mathrm{D}$ \\
$\mathrm{R}$ & $11,620 \pm 0,200 \mathrm{~B}$ & $10,220 \pm 0,160 \mathrm{DE}$ & $10,350 \pm 0,100 \mathrm{D}$ & $11,950 \pm 0,110 \mathrm{~A}$ & $11,960 \pm 0,090 \mathrm{~A}$ & $10,870 \pm 0,090 \mathrm{C}$ & $10,110 \pm 0,070 \mathrm{E}$ \\
$\phi$ & $2,643 \pm 0,001 \mathrm{~B}$ & $2,563 \pm 0,018 \mathrm{D}$ & $2,548 \pm 0,007 \mathrm{D}$ & $2,610 \pm 0,013 \mathrm{BC}$ & $2,715 \pm 0,015 \mathrm{~A}$ & $2,698 \pm 0,026 \mathrm{~A}$ & $2,603 \pm 0,015 \mathrm{C}$ \\
\hline \multicolumn{7}{c}{ Temperatura média superior } \\
$\mu$ & $24,040 \pm 0,130 \mathrm{~A}$ & $22,740 \pm 0,050 \mathrm{BC}$ & $22,900 \pm 0,170 \mathrm{~B}$ & $23,950 \pm 0,120 \mathrm{~A}$ & $22,210 \pm 0,370 \mathrm{D}$ & $24,230 \pm 0,190 \mathrm{~A}$ & $22,440 \pm 0,140 \mathrm{CD}$ \\
$\mathrm{R}$ & $11,860 \pm 0,200 \mathrm{~B}$ & $10,130 \pm 0,200 \mathrm{~F}$ & $10,800 \pm 0,110 \mathrm{D}$ & $12,150 \pm 0,230 \mathrm{~A}$ & $11,800 \pm 0,140 \mathrm{~B}$ & $11,340 \pm 0,220 \mathrm{C}$ & $10,500 \pm 0,100 \mathrm{E}$ \\
$\phi$ & $2,513 \pm 0,009 \mathrm{C}$ & $2,460 \pm 0,007 \mathrm{D}$ & $2,485 \pm 0,009 \mathrm{CD}$ & $2,529 \pm 0,013 \mathrm{BC}$ & $2,610 \pm 0,030 \mathrm{~A}$ & $2,577 \pm 0,029 \mathrm{AB}$ & $2,513 \pm 0,023 \mathrm{C}$ \\
\hline
\end{tabular}

Médias seguidas de letras distintas nas linhas diferem entre si pelo teste $t(p<0,05)$

As temperaturas da superfície inferior das telhas foram muito parecidas ao longo do dia (Figura 7). Fiorelli et al. (2009) observaram aumento no valor da carga térmica de radiação ao longo do dia, para todas as coberturas com telha de cerâmica, cerâmica pintada de branco, reciclada e fibrocimento, atingindo valor máximo às $14 \mathrm{~h}$.

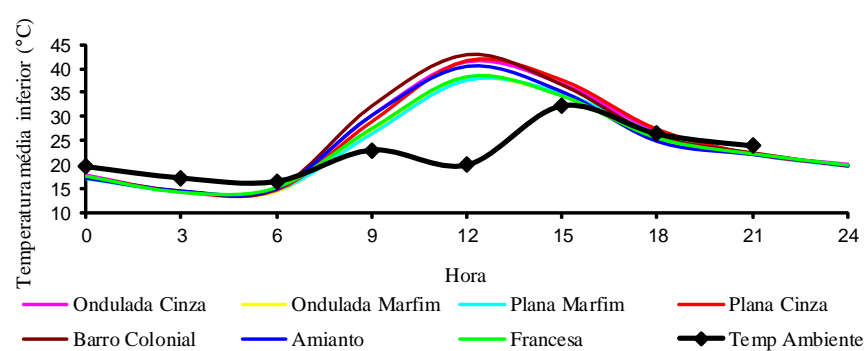

Figura 7. Temperatura média da superfície inferior da telha em função do horário

Nos horários das 9, 12 e 15 h, todas as telhas apresentaram temperatura média da superfície inferior maior que a temperatura ambiente. A partir das $15 \mathrm{~h}$ a temperatura da superfície inferior das telhas diminui em relação à temperatura ambiente, até as 6 horas. A temperatura média da superfície superior das telhas teve o mesmo comportamento cíclico da temperatura média da superfície inferior das telhas (Figura 8).

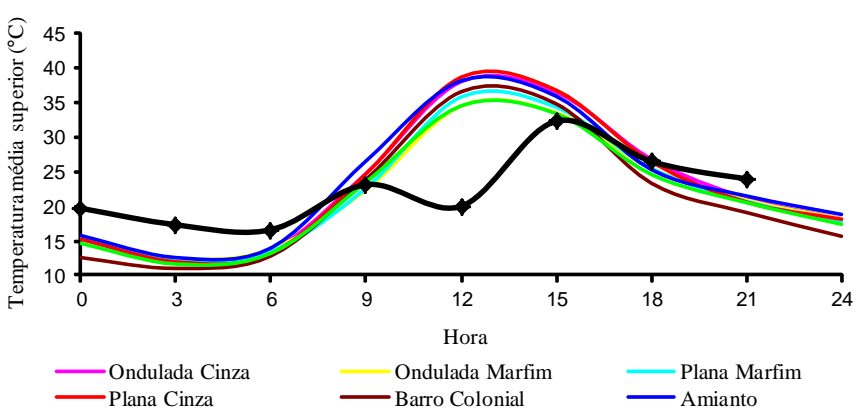

Figura 8. Temperatura média da superfície superior da telha, em função do horário

Durante o período de coleta dos dados verificou-se, às 6 $\mathrm{h}$, que algumas telhas apresentaram condensação. Condensação é uma das fases em que ocorre a transformação da matéria, do estado gasoso para o estado líquido; desta forma, o ar quente e úmido em contato com uma superfície fria se condensa.
Este é um fenômeno indesejável no interior das instalações de aves e suínos em virtude de aumentar a umidade ambiente, proliferação de doenças e, no caso de aves, diminui a qualidade da cama. A telha de barro colonial apresentou condensação na superfície superior não sendo prejudicial para os animais e a telha de amianto apresentou, tanto na superfície inferior como na superior, a condensação. Desta forma, essas telhas não seriam indicadas para uso em instalações de aves e suínos.

\section{CONCLusões}

1. As telhas com coloração marfim são melhores que as telhas com coloração cinza, nas respectivas categorias.

2. A temperatura das telhas não é alterada pela forma ondulada ou plana.

3. A telha de barro colonial tem maior temperatura da superfície inferior.

4. As telhas onduladas cinza, plana cinza e amianto, têm maiores temperaturas médias na superfície superior.

5. Os menores valores de temperatura das superfícies das telhas não significam, necessariamente, conforto térmico dos animais, condição esta que deve ser confirmada medindo-se a carga térmica de radiação recebida pelos animais sob essas coberturas.

\section{LITERATURA CITADA}

Abreu, P. G.; Abreu, V. M. N; Dalla Costa, O. A. Avaliação de coberturas de cabanas de maternidade em sistema intensivo de suínos criados ao ar livre (Siscal), no verão. Revista Brasileira de Zootecnia. v.30, p.1728-1734. 2001.

Akbari, H.; Levinson, R.; Rainer, L. Monitoring the energy-use effects of cool roofs on California commercial buildings. Energy and Buildings, v.37, p.1007-1016, 2005.

D’Orazio, M.; Di Pernax, C.; Di Giuseppea, E. The effects of roof covering on the thermal performance of highly insulated roofs in Mediterranean climates. Energy and Buildings, v.42, p.1619-1627, 2010.

Faghih, A. K.; Bahadori, M. N. Solar radiation on domed roofs. Energy and Buildings. v.41, p.1238-1245, 2009.

Faghih, A. K; Bahadori, M. N. Three dimensional numerical investigation of air flow over domed roofs. Journal of Wind Engineering and Industrial Aerodynamics. v.98, p.161-168. 2010. 
Fiorelli, J.; Morceli, J. A. B.; Vaz, R. I.; Dias, A. A. Avaliação da eficiência térmica de telha reciclada à base de embalagens longa vida. Revista Brasileira de Engenharia Agrícola e Ambiental, v.13, p.204-209, 2009.

Furtado, D. A.; Azevedo, P. V. de; Tinôco, I. de F. F. Análise do conforto térmico em galpões avícolas com diferentes sistemas de acondicionamento. Revista Brasileira de Engenharia Agrícola e Ambiental, v.7, p. 559-564. 2003.

Liberati, P.; Zappavigna, P. A dynamic computer model for optimization of the internal climate in swine housing design. Transactions of the ASABE, v.50, p.2179-2188, 2007.

Moraes, S. R. P.; Tinôco, I. F. F.; Baeta, F. C.; Cecon, P. R. Conforto térmico em galpões avícolas, sob coberturas de cimento-amianto e suas diferentes associações. Revista Brasileira de Engenharia Agrícola e Ambiental, v.3, p.89-92, 1999.

Morettin, P. A.; Toloi, C. M. C. Análise de séries temporais. São Paulo: Edgar Blücher, 2004. 535p.

Rosa, Y. B. C. J. Influência de três materiais de cobertura no índice de conforto térmico em condições de verão, para Viçosa - MG. Viçosa: UFV, 1984. 77p. Dissertação Mestrado
Santos, R. C.; Tinoco, I. F. F.; Paulo, M. O. de; Cordeiro, M. B.; Silva, J. N. Análise de coberturas com telhas de barro e alumínio, utilizadas em instalações animais para duas distintas alturas de pé-direito. Revista Brasileira de Engenharia Agrícola e Ambiental, v.6, p.142-146, 2002.

Sarmento, L. G. V.; Dantas, R. T.; Furtado, D. A.; Nascimento, J. W. B.; Silva, J. H. V. Efeito da pintura externa do telhado sobre o ambiente climático e o desempenho de frangos de corte. Agropecuária Técnica. v.26, p.117-122, 2005.

SAS Institute Inc. SAS User's guide: Statistics. Version 9.1. Cary: SAS, 2003. 176p.

Tanga, R.; Etzionb, Y. Cooling performance of roof ponds with gunny bags floating on water surface as compared with a movable insulation. Renewable Energy, v.30, p.1373-1385, 2005.

Turnpenny, J. R.; Wathes, C. M.; Clark, J. A.; McArthur, A. J. Thermal balance of livestock. 2. Applications of a parsimonious model. Agricultural and Forest Meteorology, v.101, p.29-52, 2000.

Wray, C.; Akbari, H. The effects of roof reflectance on air temperatures surrounding a rooftop condensing unit. Energy and Buildings, v.40, p.11-28, 2008. 